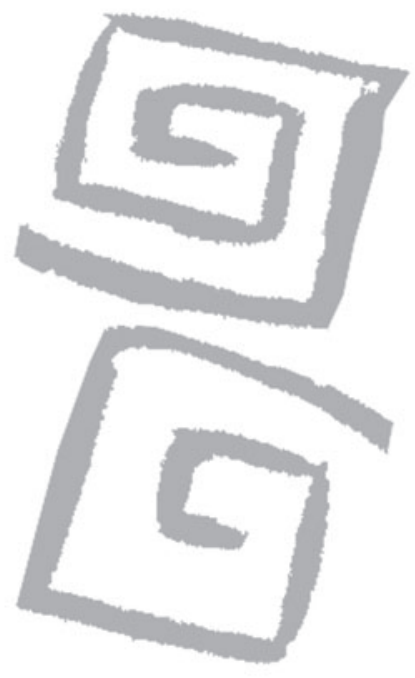

\title{
Origen y calidad del agua para consumo humano: salud de la población residente en el área de la cuenca Matanza-Riachuelo del Gran Buenos Aires
}

\author{
The origin and quality of water for human consumption: \\ the health of the population residing in the Matanza- \\ Riachuelo river basin area in Greater Buenos Aires
}

Monteverde, Malena ${ }^{1}$; Cipponeri, Marcos ${ }^{2}$; Angelaccio, Carlos ${ }^{3}$; Gianuzzi, Leda ${ }^{4}$

'Licenciada en Economía. Doctora en Economía con especialización en Estadística y Econometría. Investigadora CONICET, Centro de Investigaciones y Estudios sobre Cultura y Sociedad (CIECS-CONICET), Universidad Nacional de Córdoba, Argentina. montemale@yahoo.com

${ }^{2}$ Ingeniero Hidráulico. Director, Unidad de Investigación Desarrollo y Docencia-Gestión Ambiental (UIDD-GA). Profesor Adjunto Ordinario, Universidad Nacional de La Plata,

Argentina.

mcipponeri@ing.unlp.edu.ar

${ }^{3}$ Ingeniero Hidráulico. Consultor Naciones Unidas. Asesor, Unidad de Investigación Desarrollo y Docencia-Gestión Ambiental (UIDD-GA), Universidad

Nacional de La Plata, Argentina.

angelaccio@ciudad.com.ar

${ }^{4}$ Doctora en Ciencias Químicas. Profesora de la Cátedra de Toxicología, Facultad de Ciencias Exactas, Universidad Nacional de La Plata. Investigadora Principal CONICET, Centro de Investigación y Desarrollo en Criotecnología de Alimentos (CIDCA-CONICET),

Universidad Nacional de La Plata, Argentina.

leda@biol.unlp.edu.ar
RESUMEN El objetivo del estudio es analizar el origen y la calidad del agua domiciliaria utilizada para el consumo, en una muestra de hogares del área de la cuenca Matanza-Riachuelo del Gran Buenos Aires, Argentina. Según su origen, el 9\% de las muestras de agua provenientes de la red pública, el $45 \%$ de las de agua envasada y el $80 \%$ de las provenientes de perforaciones o pozos individuales resultaron no potables por exceso de coliformes, Escherichia coli o nitratos. Los individuos de la muestra de hogares en los que la fuente principal de agua para el consumo eran pozos individuales presentaron una probabilidad 55\% superior de padecer alguna enfermedad de origen hídrico, probabilidad que llegaría al 87\% en el caso de las diarreas y al $160 \%$ en el de las dermatitis. El agua para consumo humano en este territorio debería provenir de fuentes centralizadas que aseguren el control de la calidad del agua.

PALABRAS CLAVES Ambiente; Calidad del Agua; Enfermedades Transmitidas por el Agua; Argentina.

ABSTRACT The aim of this study is to analyze the origin and quality of water used for consumption in a sample of households in Matanza-Riachuelo river basin area in Greater Buenos Aires, Argentina. The results of drinking water by source indicated that $9 \%$ of water samples from the public water system, $45 \%$ of bottled water samples and $80 \%$ of well water samples were not safe for drinking due to excess content of coliforms, Escherichia coli or nitrates. Individuals living in households where well water is the main source of drinking water have a 55\% higher chance of suffering a water-borne disease; in the cases of diarrheas, the probability is $87 \%$ higher and in the case of dermatitis, $160 \%$ higher. The water for human consumption in this region should be provided by centralized sources that assure control over the quality of the water.

KEY WORDS Environment; Water Quality; Waterborne Diseases; Argentina. 


\section{INTRODUCCIÓN}

Las formas en que se desarrollan las áreas marginales de las ciudades, sin tener en cuenta los condicionantes del medio natural ni las necesidades en términos de infraestructura, generan una serie de problemas socioambientales derivados de la falta de agua potable, las inundaciones, las inadecuadas condiciones de higiene por falta de instalaciones sanitarias, el hacinamiento y la ausencia de patrones urbanísticos que impiden desde la limpieza pública hasta la entrada de una ambulancia, y que exponen a la población a un medio natural contaminado (1). El Gran Buenos Aires (a), una de las áreas más densamente pobladas de la Argentina, que alberga al $30 \%$ de la población total del país -más de 12 millones de personas, de acuerdo con datos del Censo Nacional de Población, Hogares y Vivienda 2010 (2) - y uno de los 12 conglomerados urbanos más grandes del mundo (3), es un ejemplo de crecimiento no planificado de población, con importantes déficits en servicios de saneamiento básico, y de la existencia de numerosos factores de riesgo ambiental a los que se ven expuestos sus habitantes.

De acuerdo a estimaciones de Cravino et al. (4), el crecimiento poblacional en las villas y asentamientos de los 24 partidos de la provincia de Buenos Aires (que forman parte del Gran Buenos Aires) ha sido considerable y con una tendencia creciente en los últimos años. El crecimiento de la población en ese tipo de asentamientos informales durante el período 1981-2001 fue del $63 \%$ en el partido de Merlo y del $86 \%$ en el partido de Moreno, mientras que en Almirante Brown, Esteban Echeverría y Lomas de Zamora el crecimiento alcanzó el $1.800 \%$, el $666 \%$ y el $228 \%$, respectivamente (solo por mencionar los cambios más importantes). Por su parte, en el mismo trabajo se muestra que la población que vive en asentamientos informales ha crecido mucho más rápido que la población total. Entre 1981 y 2006, la población en villas y asentamientos creció en términos relativos un $220 \%$ frente a un $35 \%$ de incremento poblacional en el Gran Buenos Aires. Se estima que, en los cinco años que van desde el censo 2001 hasta 2006, por cada 100 nuevos habitantes de los 24 partidos del Gran Buenos Aires, 60 se ubicaron en asentamientos informales y 40 en la ciudad "formal", mientras que la proporción de población que se ubicó en asentamientos informales era de 10 cada 100 en el período 1981-1991 y de 26 cada 100 entre 1991 y 2001 (4).

Lvovsky (5) estima que alrededor del 5,5\% de la pérdida de los años de vida ajustados por discapacidad en América Latina y el Caribe tiene su origen en las deficiencias de los servicios de saneamiento, en comparación con el $1 \%$ en los países industrializados.

Por su parte, la Organización Mundial de la Salud indica que la falta de agua segura, de servicios sanitarios y de higiene constituye el factor de riesgo ambiental más importante a nivel mundial en términos de años de vida ajustados por discapacidad y el segundo factor de riesgo más importante en términos de muertes (6).

Una evaluación de los impactos que tendría la provisión de servicios de saneamiento indica que, si el acceso a los servicios fuera universal, el número mundial de episodios de diarrea se reduciŕa en promedio un $16,7 \%$. Si se suma la desinfección del agua en el lugar de consumo, la reducción de los episodios alcanzaría en promedio el $53 \%$. Por último, proporcionar acceso a un abastecimiento regulado de agua corriente, conexión en la vivienda a la red de cloacas y tratamiento parcial de las aguas residuales, permitiría conseguir una reducción promedio del $69 \%$ (7).

De acuerdo con los datos del censo de población del año 2010 (2), la cobertura de los servicios de red pública de agua y red cloacal en los 24 partidos del Gran Buenos Aires es marcadamente heterogénea, con niveles que oscilan entre el $10 \%$ (en el partido de Malvinas Argentinas) y el 100\% (en Vicente López) para los hogares que cuentan con agua proveniente de la red pública (b), y con cobertura de la red cloacal que oscila entre el 2\% (en Malvinas Argentinas) y el 97\% (en Vicente López). De acuerdo a la misma fuente, la cobertura promedio para toda la provincia de Buenos Aires de la red pública de agua sería del $75 \%$ y la de la red cloacal no alcanzaría a servir a la mitad de los hogares (el $48 \%$ ). Dichas cifras contrastan fuertemente con los niveles de CObertura en la Ciudad Autónoma de Buenos Aires, con el $100 \%$ y el $98 \%$ de los hogares con acceso a cada uno de los servicios, respectivamente. 
Los resultados de un estudio reciente sobre los partidos del Gran Buenos Aires sugieren la existencia de un fuerte efecto por la falta de servicios de saneamiento básico sobre la probabilidad de padecimiento de diarreas, así como la existencia de un importante efecto sinérgico entre la falta de servicios de saneamiento y el nivel de educación del jefe del hogar y la cercanía a basurales que incrementa sustancialmente el riesgo de padecimiento de dicha enfermedad (8).

El objetivo del presente estudio es profundizar en el análisis de la relación entre el déficit de servicios de saneamiento (o el déficit en su calidad) y el padecimiento de enfermedades hidrotransmisibles entre la población residente en el área de la cuenca Matanza-Riachuelo (c) (perteneciente al Gran Buenos Aires). Para ello se analiza el origen y la calidad del agua utilizada para el consumo en una muestra de hogares del área de estudio en los que previamente se detectó el padecimiento de alguna enfermedad de origen hídrico, y se estudia la correlación de los resultados con la presencia de cada una de las enfermedades objeto de estudio.

\section{MÉTODOS}

Para estudiar en qué medida la baja calidad del agua que se utiliza en los hogares sería el mecanismo que incrementa el riesgo de padecimiento de enfermedades de origen hídrico entre la población sin acceso a agua proveniente de la red pública, entre octubre de 2010 y febrero de 2011 se recogieron muestras de agua en 90 hogares localizados en la cuenca Matanza-Riachuelo donde habitaban 380 personas.

La muestra maestra estuvo constituida por 151 hogares en los que se detectaron casos de individuos que padecieron alguna enfermedad de origen hídrico durante el último año (mediante autoreporte de salud) a través de una encuesta realizada entre mayo y septiembre de 2010 en los partidos de la cuenca Matanza-Riachuelo. Dicha encuesta se basó en un diseño muestral aleatorio, bietápico estratificado dentro del territorio de la cuenca Matanza-Riachuelo. La unidad de muestreo de la primera etapa fueron radios censales y la unidad de muestreo de la segunda etapa fueron viviendas. Los criterios de estratificación (de la primera etapa) fueron tres: 1) el acceso o no a agua de red y a conexión a la red cloacal, 2) áreas inundables y no inundables y 3) un indicador sintético de vulnerabilidad de la población (en este caso se trabajó con necesidades básicas insatisfechas). Se seleccionaron 40 radios censales de forma aleatoria dentro de cada estrato y se realizaron 20 encuestas por cada radio (seleccionando viviendas de forma aleatoria).

Para minimizar los problemas de estacionalidad de las enfermedades hídricas, el período de referencia de las preguntas sobre dichas enfermedades fueron los últimos doce meses.

La muestra final para la encuesta de 2011 quedó constituida por aquellos hogares pertenecientes a la muestra maestra en los que se reportaron enfermedades de origen hídrico en el último año y en los que se pudo acordar una cita previa (130 hogares). De ellos, 90 hogares fueron los que atendieron al personal de campo (la persona a cargo de tomar la muestra de agua y la persona a cargo de administrar el cuestionario de preguntas complementarias). La razón más frecuente de rechazo aludida por los hogares estuvo asociada a cuestiones de seguridad (aun en los casos en los que se había acordado la entrevista telefónicamente). Sin embargo, los casos de no respuesta no siguieron un patrón geográfico ni estuvieron relacionados con las características del hogar.

Se obtuvieron muestras de agua en hogares de los partidos de Almirante Brown, Avellaneda, Cañuelas, Esteban Echeverría, La Matanza, Lanús, Lomas de Zamora, Marcos Paz y de la Ciudad Autónoma de Buenos Aires, en sectores servidos por servicios centralizados (agua de red) o individuales (agua de pozo o perforación).

Las muestras se obtuvieron de la canilla utilizada para la provisión de agua para consumo del hogar (alimentada directamente desde la fuente o desde el sistema de almacenamiento -tanque o cisterna- de la vivienda). Además, se evaluó la conveniencia de tomar muestras adicionales en aquellos casos en los que había dudas sobre el estado sanitario del tanque de agua. En dichos casos se buscó tomar la muestra en la acometida de la fuente, antes del tanque. En los casos en que la fuente principal de consumo para bebida del hogar resultara agua envasada, se tomó una muestra del dispenser de la misma. De este modo, en 66 hogares se tomó una muestra de agua, en 
21 hogares se tomaron dos muestras y en 3 se obtuvieron tres muestras $y$, por tanto, en total se tomaron 117 muestras en 90 hogares.

Conjuntamente con la toma de las muestras de agua, se administró un cuestionario de preguntas orientadas a indagar acerca de los siguientes aspectos: el origen del agua para el hogar, la existencia de tanque elevado de agua, el control periódico del tanque y si tenía tapa. Además, entre los hogares en los que se detectó que obtenían el agua principalmente de perforación o pozo, se les preguntó a qué profundidad se construyó, la antigüedad, si la perforación contaba con camisa y filtro, la fecha de realización del último análisis de la calidad de agua del pozo y el costo del análisis, y se les pidió que hicieran una evaluación personal de la calidad del agua que se obtenía de dicho pozo y que justifique su opinión sobre esa evaluación. Por su parte, a todos los encuestados se les preguntó si aplicaban algún tratamiento al agua para beber o preparar comida y si compraban agua envasada. Además, se hicieron una serie de preguntas orientadas a conocer el sistema de desagüe de aguas servidas con que contaba la vivienda, si el pozo absorbente está cerca del sitio de extracción del agua, la frecuencia de desagote y la vida útil del pozo.

Los procedimientos seguidos para la obtención y análisis de las muestras de agua se realizaron según los Standard Methods for the Examination of Water and Wastewater (9). Las muestras de agua fueron tomadas en recipientes estériles de $250 \mathrm{ml}$ y trasladadas al laboratorio en forma refrigerada según los métodos 9060 y 9060B (9). Las muestras de agua de red que contenían cloro fueron neutralizadas con solución estéril de tiosulfato de sodio $0.1 \mathrm{~N}$ antes de realizar los ensayos microbiológicos. Las determinaciones microbiológicas se realizaron siguiendo el procedimiento descripto por Eaton et al. (9). Para recuento de coliformes totales se empleó el método 9221 y para presencia de Escherichia coli el método 9225.

Además de los análisis de coliformes fecales, coliformes totales y de Escherichia coli, para una submuestra de 34 hogares se realizó además análisis de contenido de nitratos (d). La determinación de nitrato se realizó siguiendo el procedimiento descripto en Standard Methods 4500- $\mathrm{NO}_{3}{ }^{-}$Nitrogen (Nitrate) (9).
La muestra se consideró no potable cuando los coliformes totales resultaron mayores a 2 NMP/100ml, el test para Escherichia coli resultó positivo o la concentración de nitratos resultó mayor a $35 \mathrm{mg} / \mathrm{l}$ (e).

El resultado del análisis bacteriológico y de nitratos de las muestras de agua, conjuntamente con la información obtenida mediante el cuestionario de preguntas, se cargó al programa Statistical Package for the Social Sciences (SPSS) versión 15.0. Dicha base de datos fue consistida y anexada a una base de datos más amplia (desde donde se obtuvo el marco muestral para la obtención de muestras de agua, basado en la existencia de antecedentes de enfermedades hidrotrasmisibles), lo que permitió incorporar a la base inicial información adicional tanto a nivel de individuos como de hogares. La información adicional a nivel de individuos consistió en: la edad, el sexo, el nivel de educación, la cobertura de salud, la situación laboral y el autoreporte de padecimiento de enfermedades de origen hídrico de cada miembro del hogar (las enfermedades contempladas fueron: diarreas, hepatitis, dermatitis, infecciones intestinales, gastroenteritis, parásitos y leptospirosis). La información adicional a nivel de hogar incluyó: información sobre el contexto ambiental de la vivienda, tipo de vivienda, materiales de la misma, cantidad de ambientes, origen y tratamiento del agua, ubicación y tipo de descarga del baño y destino del desagüe del inodoro.

La base de datos final se tradujo a una base de datos en formato Stata/SE (versión 10.1), programa que se utilizó para el análisis estadístico de la información. Este análisis consistió principalmente en el cálculo de tablas de frecuencias relativas, tablas de contingencia o de correlación, la aplicación de test estadísticos de comparación directa de proporciones (test binomial exacto) y test de diferencia de medias (test $\mathrm{t}$ de Student). Adicionalmente, se estimaron modelos de regresión con variables dependientes dicotómicas para analizar el efecto del origen y la calidad del agua sobre la probabilidad de padecer enfermedades de origen hídrico, controladas por posibles variables de confusión (como la edad, el sexo y el bajo nivel educativo). 


\section{RESULTADOS}

El total de muestras de agua recogidas y analizadas fue de 117, provenientes de 90 hogares (en algunos hogares se tomó más de una muestra, como fue mencionado en el apartado anterior).

La distribución de las muestras según el origen del agua fue la siguiente: $18 \%$ agua envasada, $35 \%$ de pozo y $47 \%$ de red. La media de edad de los individuos de la muestra fue de 32 años y el $52 \%$ fueron mujeres. Respecto al autoreporte de enfermedades de origen hídrico, se observó que un $45 \%$ de los individuos de la muestra padecieron al menos una enfermedad hidrotransmisible en el último año. Específicamente, la prevalencia de diarreas entre los individuos fue del $29 \%$, del $15 \%$ para gastroenteritis, $5 \%$ para dermatitis, infecciones intestinales y parásitos intestinales y del $3 \%$ para parásitos extraintestinales. Cabe recordar que el universo estuvo constituido por los hogares que reportaron al menos un miembro con alguna enfermedad de origen hídrico el último año y por tanto dichas prevalencias no son extrapolables a toda la población. El 30\% de los individuos no contaba con ningún tipo de seguro médico (público o privado). Del total de hogares de la muestra, el $25 \%$ contaba con jefas o jefes de hogar ( $f$ ) que no habían alcanzado la primaria completa y se observaron porcentajes elevados de hogares en los que la falta de asfalto (55\%), presencia de inundaciones frecuentes (48\%) y basurales (58\%) eran problemas del barrio. Al analizar la existencia de hogares en viviendas precarias (g), se observó un porcentaje relativamente bajo en la muestra (el $2 \%$ ), lo que refleja que las muestras no provienen de las zonas de asentamiento precario y a priori más vulnerables del Gran Buenos Aires (las denominadas villas o asentamientos informales), aunque un $60 \%$ del total de la muestra indicó que el desagüe del inodoro no descargaba a la red pública, es decir que las viviendas no contaban con conexión a la red cloacal.

Sobre la base del criterio de potabilidad detallado en la sección Métodos, los análisis de las muestras de agua arrojaron los siguientes resultados: un $40 \%$ de las muestras resultó ser "no potable" por exceso de coliformes fecales, totales y/o Escherichia coli.
Considerando el exceso de nitratos (que fue analizado solamente para un tercio de la muestra), el porcentaje que excede los niveles aceptables asciende al 35\%. En el análisis conjunto de coliformes, Escherichia coli y nitratos, el porcentaje de muestras de agua que califican como "no potables" alcanzó al 44\%.

La representación gráfica de los resultados de no potabilidad según el origen del agua se aprecian en la Figura 1 (para coliformes fecales, totales y Escherichia coli) y en la Figura 2 (para nitratos).

La Figura 1 muestra que el 9\% de las muestras de agua provenientes de red resultaron "no potables" según resultados bacteriológicos, mientras que dicho porcentaje resultó del $45 \%$ para las muestras de agua envasada y del $80 \%$ para las muestras de agua provenientes de pozos individuales. Aunque las diferencias según el origen del agua son evidentes, se aplicó un test estadístico de comparación de proporciones que arrojó que las diferencias en todos los casos son estadísticamente significativas al $1 \%$.

Por su parte, la Figura 2 muestra que ninguna de las muestras de agua envasada (en total 5) excedió los límites aceptables de nitratos, el 12\% de las muestras de agua de red contenían exceso de nitratos y el $83 \%$ de las muestras de agua provenientes de pozo individual excedían dicho límite.

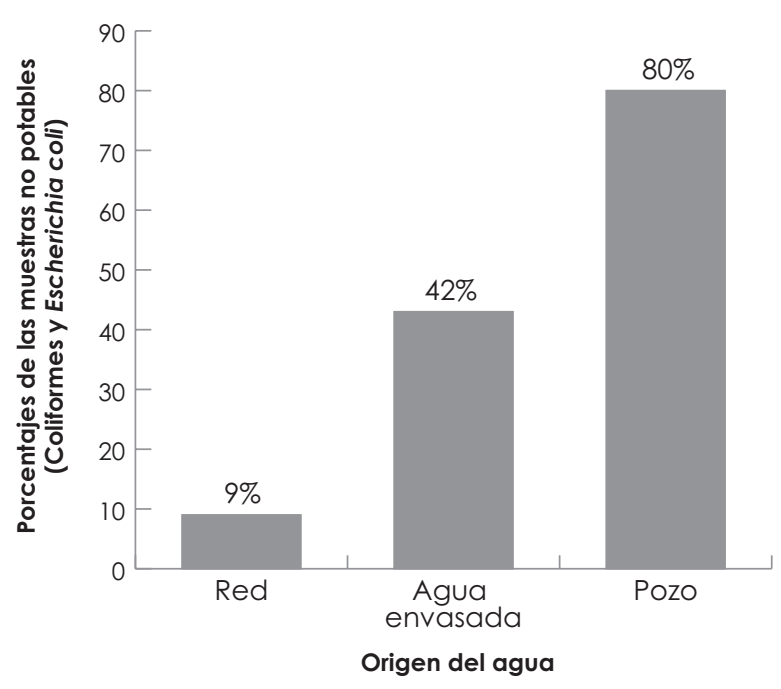

Figura 1. Porcentajes de muestras de agua no potable por coliformes fecales y Escherichia coli, según su origen. Cuenca Matanza-Riachuelo, 2011.

Fuente: Elaboración propia a partir de datos primarios. 


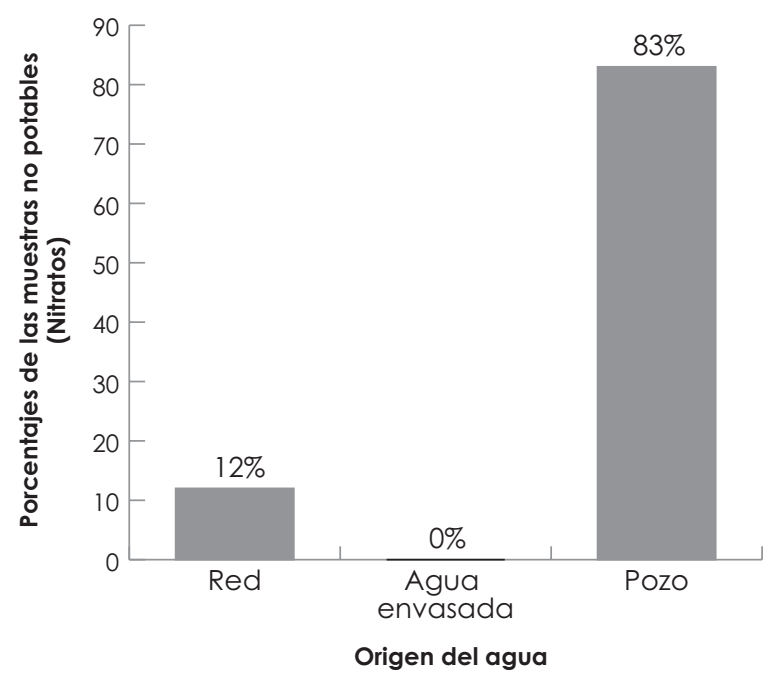

Figura 2. Porcentajes de muestras de agua no potable por nitratos, según su origen. Cuenca Matanza-Riachuelo, 2011.

Fuente: Elaboración propia a partir de datos primarios.

El total de las muestras de agua de red que resultaron no potables de acuerdo al estudio bacteriológico, son muestras que fueron colectadas luego de haber sido almacenadas en tanques o haber atravesado algún tipo de filtro (no muestras directas de la red colectadas antes de pasar por el tanque o algún sistema individual de filtrado). Sin embargo, el $8 \%$ de las muestras de agua de red directa (2 casos de 26) resultaron no potables por exceso de nitratos.

Respecto a las muestras de agua provenientes de pozos individuales, no se observaron diferencias significativas en los porcentajes de muestras no potables según se trate de muestras provenientes de pozo directo o de agua de pozo que se almacena en tanque. En el primer caso (muestras no potables del total de muestras de agua de pozo directo) el porcentaje fue del $20 \%$ $y$, en el segundo (muestras no potables del total de muestras de agua de pozo que se almacena en tanque) fue del $19 \%$.

$\mathrm{Al}$ analizar las respuestas de las encuestas respecto de la profundidad de los pozos individuales de extracción de agua, y adoptando como valor singular de contraste la profundidad de 40 metros (donde existe para la región una alta probabilidad de encontrar el techo del acuífero Puelches y por ende las aguas subterráneas de mejor calidad) se pueden realizar las siguientes observaciones:
- Se tomaron 27 muestras de agua de pozo de las cuales 6 resultaron negativas para los parámetros bacteriológicos (22\%) y 21 positivas (78\%).

- De las 6 que resultaron negativas, 4 provenían de pozos de profundidad mayor a 40 metros $(67 \%)$ y dos a profundidad menor a esa cifra (33\%).

- De las 21 muestras de agua positivas, 10 provenían de pozos de profundidades mayores a 40 metros $(48 \%)$ y 11 de pozos menores a 40 metros $(52 \%)$.

En cuanto a la presencia de nitratos en pozos donde se conocía su profundidad, de 5 pozos de menos de 40 metros de profundidad, 4 superaron el límite permitido para nitratos $(80 \%)$ y 1 no. De los 3 pozos de más de 40 metros de profundidad las 3 muestras superaron el límite permitido para nitratos $(100 \%)$.

Dos pozos más superaron el límite permitido para nitratos sin conocerse su profundidad. En resumen, de las 11 muestras que se obtuvieron de pozos individuales en las que se analizaron nitratos, 10 resultaron "no potables" y 1 no excedió los niveles de nitrato, independientemente de la profundidad del pozo.

Los resultados expuestos en los dos párrafos anteriores muestran que el agua proveniente de pozos individuales es mayoritariamente no potable, sin importar la profundidad del pozo. Ello pone de manifiesto dos cuestiones básicas: por un lado, muestra el grado de deterioro de origen antrópico (caracterizado por la presencia de nitratos) del acuífero semiconfinado Puelche, históricamente de buena calidad y poco vulnerable. Por otro lado, plantea dudas sobre la eficiencia del aislamiento, entre acuíferos contaminados y no contaminados, alcanzado en las perforaciones individuales (tanto domiciliarias como industriales).

Resultó significativa la diferencia entre las muestras negativas y positivas (análisis bacteriológicos) en la proporción de hogares en los que se reportó que el pozo contaba con camisa y filtro. Para las primeras (las que no excedieron los niveles aceptables), el $67 \%$ de los pozos contaría con camisa y filtro, mientras que para las muestras "no potables" dicho porcentaje sería del $48 \%$. A pesar de que esta diferencia resultó significativa, la elevada proporción de falta de respuesta a esta pregunta hace que se deba tomar con cautela el resultado y se requiera de mayores estudios para extraer conclusiones. 
Para evaluar en qué medida el origen del agua representa un riesgo para la salud (dentro del grupo poblacional incluido en la muestra), se estimaron un conjunto de modelos de regresión logística a nivel de individuos cuyas variables dependientes son iguales a uno en caso de autoreporte positivo de enfermedad.

El Cuadro 1 presenta las razones de posibilidad de los parámetros de interés con su significación estadística respectiva (valor de p) para los modelos logísticos de cada una de las enfermedades. Entre las variables dependientes se incluye el haber padecido "al menos alguna de las enfermedades de origen hídrico" y el resto corresponden a las enfermedades específicamente contempladas en el estudio y para las cuales se reportaron casos, ellas son: diarreas, gastroenteritis, infecciones intestinales, parásitos intestinales y dermatitis. La variable independiente objeto de estudio, es "origen del agua", que corresponde a una variable dicotómica con valor uno si el origen principal del agua utilizada para consumo en el hogar proviene de pozo individual y cero en los otros casos (agua de red pública o agua envasada). Las variables de control son la edad, el sexo y una variable dicotómica con valor uno para las personas de 15 años y más que no tengan la primaria finalizada.
Los resultados muestran que el origen del agua (de pozo) tiene un efecto positivo y estadísticamente significativo sobre la probabilidad de padecimiento de alguna enfermedad de origen hídrico y específicamente sobre la probabilidad de padecer diarreas, infecciones intestinales y dermatitis. Para este mismo origen, en el caso de gastroenteritis y parásitos intestinales, las razones de posibilidad son mayores a uno, pero no resultan estadísticamente significativos.

La magnitud de las razones de posibilidad estimadas indican que:

- Los individuos que viven en hogares en los que el agua de pozo individual es la fuente principal de agua para consumo, tienen una probabilidad $55 \%$ superior de padecer alguna enfermedad de origen hídrico que aquellos que viven en hogares cuya fuente principal es el agua de red o el agua envasada.

- Dicho incremento de probabilidad sería del $87 \%$ en el caso de diarreas y del $160 \%$ en el caso de dermatitis.

Cabe resaltar que la variable "origen del agua" resulta estadísticamente significativa en los casos mencionados, aún después de controlar por una variable proxy del estatus socioeconómico como es el nivel educativo y por variables demográficas (la edad y el sexo).

Cuadro 1. Estimaciones logísticas de la probabilidad de padecimiento de enfermedades de origen hídrico con "origen del agua" como variable explicativa (estimación robusta de la varianza). Cuenca Matanza-Riachuelo, 2011.

\begin{tabular}{|c|c|c|c|c|c|c|c|c|}
\hline \multirow{3}{*}{$\begin{array}{l}\text { Enfermedades de origen } \\
\text { hídrico }\end{array}$} & \multicolumn{8}{|c|}{ Variables explicativas } \\
\hline & \multicolumn{2}{|c|}{ Edad } & \multicolumn{2}{|c|}{$\operatorname{Sexo}^{a}$} & \multicolumn{2}{|c|}{ Origen del aguab } & \multicolumn{2}{|c|}{ Primaria incompleta } \\
\hline & $\begin{array}{l}\text { Razón de } \\
\text { posibilidad }\end{array}$ & $\begin{array}{l}\text { Valor } \\
\text { de } p\end{array}$ & $\begin{array}{l}\text { Razón de } \\
\text { posibilidad }\end{array}$ & $\begin{array}{l}\text { Valor } \\
\text { de p }\end{array}$ & $\begin{array}{l}\text { Razón de } \\
\text { posibilidad }\end{array}$ & $\begin{array}{l}\text { Valor } \\
\text { de p }\end{array}$ & $\begin{array}{l}\text { Razón de } \\
\text { posibilidad }\end{array}$ & $\begin{array}{l}\text { Valor } \\
\text { de } p\end{array}$ \\
\hline $\begin{array}{l}\text { Algunas enfermedades de } \\
\text { origen hídrico }\end{array}$ & 1,00 & 0,90 & 1,03 & 0,75 & 1,55 & 0,06 & 1,76 & 0,03 \\
\hline Diarreas & 1,01 & 0,04 & 0,93 & 0,35 & 1,87 & 0,01 & 1,35 & 0,29 \\
\hline Gastroenteritis & 0,99 & 0,23 & 0,96 & 0,51 & 1,16 & 0,63 & 0,86 & 0,67 \\
\hline Infecciones intestinales & 1,01 & 0,14 & 0,97 & 0,78 & 0,25 & 0,06 & 1,66 & 0,35 \\
\hline Parásitos intestinales & 0,97 & 0,09 & 1,14 & 0,19 & 1,90 & 0,22 & 1,08 & 0,89 \\
\hline Dermatitis & 1,00 & 0,66 & 0,94 & 0,70 & 2,60 & 0,04 & 0,86 & 0,80 \\
\hline \multicolumn{9}{|c|}{ Fuente: Elaboración propia a partir de datos primarios. } \\
\hline
\end{tabular}




\section{DISCUSIÓN}

La falta de servicios de saneamiento básico (conexiones a la red pública de agua y de cloaca, específicamente) ha sido ampliamente mencionado como factor de riesgo para la salud. Diversos estudios demuestran que la situación de falta de cobertura de este tipo de servicios es crítica para el padecimiento de enfermedades hidrotrasmisibles en distintas regiones del mundo $y$, en algunos casos, cuantifican los efectos de dicho déficit en términos de salud o en términos de su impacto económico (5-7,10-17).

Los partidos del Gran Buenos Aires presentan déficits en servicios de saneamiento que sorprende, tanto por su magnitud, como por el hecho de encontrarse a pocos kilómetros de una de las áreas con mayor cobertura de servicios de saneamiento (entre otros) de la Argentina, la Ciudad Autónoma de Buenos Aires (CABA). Sin embargo, faltan trabajos formales que analicen la relación entre el origen del agua para consumo de los hogares, su calidad efectiva y el padecimiento de enfermedades de origen hídrico en esta área de la Argentina.

Entre las causas de la falta de cobertura de este tipo de servicios cabe mencionar las características del crecimiento urbano del Gran Buenos Aires. En este sentido el trabajo de Cravino et al. (4) resulta muy revelador: en el Gran Buenos Aires la población que vive en asentamientos informales ha estado creciendo mucho más rápido que la población total. Entre 1981 y 2006 la población en villas y asentamientos creció en términos relativos $220 \%$ frente a un $35 \%$ de incremento poblacional del área. Por razones logísticas y de seguridad en las tareas de campo, la muestra sobre la que se trabajó no contempló asentamientos informales, razón por la cual los resultados obtenidos en el presente estudio pueden considerarse conservadores.

Un estudio realizado recientemente para los 17 partidos del primer anillo del Gran Buenos Aires (los partidos más cercanos a la CABA), muestra que más del $30 \%$ de la población percibe que la salud, la falta de pavimento y las inundaciones son problemas prioritarios en su barrio, y más del 50\% que lo son los basurales y el desempleo (8). En el mismo estudio, basado en una muestra de 809 hogares en los que habitaban 3.038 individuos, se analizó además el efecto de la falta de servicios de saneamiento sobre la probabilidad de padecer diarreas, así como la interacción entre la falta de servicios y el bajo nivel educativo de la jefa o del jefe de hogar y otras variables de vulnerabilidad social y ambiental (como la cercanía a basurales). Los resultados en este sentido son claros: la falta de servicios de saneamiento (agua de red y cloacas) incrementan de forma significativa el riesgo de padecimiento de diarreas, y dicho efecto se potencia cuando se trata de grupos especialmente vulnerables (efecto de interacción positivo y elevado entre la población de bajo nivel educativo y que vive cerca de basurales).

El presente estudio buscó ahondar en los mecanismos que pueden estar incidiendo en las relaciones anteriores y en particular en qué medida el origen del agua puede ser un factor de riesgo para la salud.

El principal resultado de este estudio es la alta proporción de muestras de agua que no resultaron aptas para consumo por exceso de coliformes, Escherichia coli o nitratos (un $44 \%$ del total de la muestra), así como las diferencias en la distribución según el origen de las mismas: el 9\% de las muestras de agua provenientes de la red pública resultaron no potables, mientras que dicho porcentaje resultó del $45 \%$ para las muestras de agua envasada y del $80 \%$ para las muestras de agua provenientes de pozo individual.

Cabe resaltar los altísimos porcentajes de muestras de agua no potables provenientes de agua de pozo individual e incluso envasada. Este resultado permite una mayor comprensión de los mecanismos que operan en la relación entre la falta de servicios de saneamiento y los mayores riesgos para la salud. La mala calidad del agua para consumo de los hogares sin acceso a red pública sería clave en esta relación, y no siempre la falta de acceso a agua de red puede ser suplida por agua envasada, ya que casi en la mitad de los casos analizados, dicha agua tampoco resulta segura para el consumo.

Cabe destacar que la alta proporción de muestras de agua envasada que no resultó apta para consumo, fue un resultado particularmente sorprendente. Este resultado pone de relieve la necesidad de extremar los controles de calidad en estos casos y la importancia de profundizar en el 
estudio del origen del agua envasada que se comercializa en el Gran Buenos Aires. Es importante aclarar aquí, que todas las muestras de agua envasada que resultaron no aptas para consumo, no identificaban la marca o si lo hacían, eran marcas poco conocidas comercialmente que no informaban sobre el origen del agua.

Las características y los cambios sufridos en las fuentes de agua para consumo del Gran Buenos Aires, ayudan a explicar las razones de la existencia de una alta proporción de hogares que utilizan fuentes de agua no segura para el consumo en la actualidad. La fuente histórica para la provisión de agua potable en el Gran Buenos Aires ha sido el acuífero semiconfinado denominado Puelche, fuente que se encuentra, en términos medios, a más de 40 metros de profundidad con un espesor variable de 20 a 90 metros en el área de estudio, caracterizado por una muy buena calidad natural del agua. Sin embargo, la misma se ha visto deteriorada como consecuencia de las incesantes intervenciones antrópicas directas e indirectas, lo que ha determinado que en la actualidad no sea una fuente de abastecimiento de agua segura $(18,19)$. Si bien a partir de de la década de 1990 comenzó un proceso de sustitución de fuentes de agua para consumo humano, pasando del acuífero Puelche a las agua del Río de la Plata con tratamiento centralizado, este proceso implicó una solución para aquella población que cuenta con red pública de agua, mientras que la proporción de población que aún obtiene agua de perforaciones individuales y colectivas directas al acuífero (ya contaminado) es aún muy elevada. Los trabajos de Auge et al. brindan una pormenorizada descripción de los acuíferos de la zona y de los aspectos relacionados con su vulnerabilidad y deterioro (18-21).
Por último, los resultados del presente estudio muestran que las enfermedades de origen hídrico más correlacionadas con el origen del agua serían las diarreas, las infecciones intestinales y las dermatitis. Para dichos casos, el incremento de probabilidad de padecimiento asociado con el uso de agua de pozo individual como fuente principal sería del $87 \%$ en el caso de diarreas y del $160 \%$ en el caso de dermatitis.

Una de las limitaciones en el análisis sobre el riesgo de padecimiento de enfermedades de origen hídrico es que no se cuenta con casos diagnosticados sino con autoreportes de enfermedades. Sin embargo, dado que el objetivo del artículo no es hacer estimaciones de prevalencia o de totales, sino analizar diferenciales entre subgrupos (en este caso, entre los que cuentan con agua de red y los que no cuentan con dicho servicio), de existir el subreporte solo afectaría los resultados si fuese mayor en uno de los grupos de comparación. De ser el caso, cabe esperar que el grupo con mayor subreporte sea el más vulnerable (por problemas de información, acceso a servicios de salud y por lo tanto falta de diagnóstico adecuado) y nuestros resultados indican justamente lo contrario: las personas sin acceso a agua de red (en general, la más vulnerable) son las que presentan mayores riesgos de padecimiento de enfermedades, sugiriendo que nuestros resultados (de estar afectados por este problema) serían conservadores.

En conclusión, basándose en la incertidumbre que genera la eficiencia en la realización de perforaciones individuales y el estado de calidad del acuífero Puelche en el área del Gran Buenos Aires, el agua para consumo humano en este territorio debería provenir de fuentes centralizadas que aseguren el control de calidad del agua distribuida por red (proveniente total o parcialmente del Río de la Plata y de los acuíferos) tratada de manera centralizada o diluida convenientemente.

\section{NOTAS FINALES}

a. El Gran Buenos Aires está constituido por la Ciudad Autónoma de Buenos Aires (capital de la República Argentina) y los 24 partidos (municipios) de la Provincia de Buenos Aires que rodean a la capital del país. b. Se incluyen los hogares con provisión de agua por cañería dentro de la vivienda o del terreno y que estén conectados a la red pública de agua.

c. La cuenca Matanza-Riachuelo comprende parte de la Ciudad de Buenos Aires y de los partidos de Almirante Brown, Avellaneda, Cañuelas, 
Esteban Echeverría, Ezeiza, General Las Heras, La Matanza, Lanús, Lomas de Zamora, Marcos Paz, Merlo y San Vicente.

d. Por una cuestión de restricción presupuestaria, para los análisis de laboratorio se siguió el criterio de analizar nitratos en 3 muestras del total de 10 muestras aproximadamente recogidas en cada salida, a todas las cuales se les realizó el análisis bacteriológico. Dicha selección de 3 cada 10 muestras se realizó de forma aleatoria aunque asegurando que se hiciera al menos un análisis de nitrato por radio censal.

e. Si bien la determinación de la potabilidad del agua sigue un protocolo más amplio que el de los parámetros indicados, existe evidencia previa que indica que dichos parámetros (bacteriológicos y de nitratos) estarían dentro de los más relevantes para analizar la potabilidad en el área bajo estudio.

f. En las fuentes argentinas se elige al jefe o jefa por la "vía del reconocimiento": es decir, el jefe de un hogar es aquella persona que es reconocida como tal por los restantes miembros de ese hogar.

g. Se consideró como vivienda precaria un concepto amplio que incluye: pieza en inquilinato, pieza en hotel/pensión, local no construido para habilitación y rancho o casilla.

\section{AGRADECIMIENTOS}

El presente trabajo fue Ilevado a cabo gracias al apoyo de la Agencia Nacional de Promoción Científica y Tecnológica del Ministerio de Ciencia, Tecnología e Innovación Productiva de la Argentina, a través de los fondos del proyecto PICT 2008-1153 (Resolución 343-09). Agradecemos a los colaboradores e integrantes de la Unidad de Investigación Desarrollo y Docencia-Gestión Ambiental (UIDD-GA) que aportaron sus conocimientos y mejores esfuerzos para el diseño de las encuestas y la implementación de los trabajos de campo.

\section{REFERENCIAS BIBLIOGRÁFICAS}

1. Clichevsky N. Pobreza y políticas urbano ambientales en Argentina. Santiago de Chile: CEPAL; 2002. (Serie Medio Ambiente y Desarrollo 49).

2. Instituto Nacional de Estadística y Censos. Censo nacional de población, hogares y viviendas 2010: Total del país y provincias: resultados definitivos. Variables seleccionadas [CD-ROM]. Buenos Aires: INDEC; 2011.

3. United Nations. World urbanization prospects: the 2011 revision [Internet]. New York: UN; 2011 [citado 15 junio 2012]. Disponible en: http://esa. un.org/unpd/wup/pdf/WUP2011 Highlights.pdf.

4. Cravino MC, Del Rio JP, Duarte JI. Magnitud y crecimiento de las villas y asentamientos en el Área Metropolitana de Buenos Aires en los últimos 25 años. [Internet]. 2008 [citado 10 agosto 2010]. Disponible en: http://www.fadu.uba.ar/ mail/difusion extension/090206 pon.pdf.

5. Lvovsky K. Health and environment. Washington DC: World Bank; 2001. (Environment Strategy Papers No 1).
6. World Health Organization. Global health risks: mortality and burden of disease attributable to selected major risks. [Internet]. 2009. [citado 10 jun 2012]. Disponible en: http://www.who.int/healthinfo/global burden disease/GlobalHealthRisks report full.pōf.

7. Hutton G, Haller L. Evaluation of the costs and benefits of water and sanitation: Improvements at the global level water, sanitation and health protection of the human environment [Internet]. Geneva: WHO; 2004 [citado 15 ago 2009]. Disponible en: http://www.who.int/water_sanitation health/wsh0404.pdf.

8. Monteverde $M$, Cipponeri $M$, Angelaccio C. Falta de servicios de saneamiento, pobreza y enfermedades de origen hídrico: El caso del conurbano bonaerense. RELAP. 2010;3(6):54-69.

9. Eaton AD, American Public Health Association, American Water Works Association, Water Environment Federation. Standard methods for the examination of water and wastewater. Washington DC: APHA, AWWA, WEF; 2005

10. Ress P. European water treaty hailed as a milestone for public health. BMJ. 2005;331(7513):368. 
11. Galiani S, Gertler P, Schargrodsky E. Water for life: the impact of the privatization of water services on child mortality [Internet]. Washington DC: Inter-American Development Bank; 2002 [citado 14 nov 2011]. Disponible en: http://www.iadb. org/res/publications/pubfiles/pubS-233.pdf.

12. Mulreany JP, Calikoglu S, Ruiz S, Sapsin JW. Water privatization and public health in Latin America. Revista Panamericana de Salud Pública. 2006;19(1):23-32.

13. Robinson $\mathrm{K}$, Infante $\mathrm{R}$, Trelles J. Material de referencia en agua, saneamiento, salud y desarroIlo: Una visión desde América Latina y el Caribe [Internet]. Lima: 2006 [citado 7 oct 2011]. Disponible en: http://www.bvsde.paho.org/bvsacg/e/ foro4/producto3.pdf.

14. Organización Panamericana de la Salud, Organización Mundial de la Salud. Informe regional sobre la evaluación 2000 en la Región de las Américas: Agua potable y saneamiento, estado actual y perspectivas [Internet]. Washington DC: OPS; 2001 [citado 25 mar 2011]. Disponible en: http://www.bvsde.opsoms.org/bvsaas/e/fulltext/infregio/infregio.pdf.

15. Maceira D, Kremer P, Finucane H. El desigual acceso a los servicios de agua corriente y cloacas en la Argentina. Políticas Públicas Análisis No 39 [Internet]. Buenos Aires: CIPPEC; 2007 [citado 25 mar 2011]. Disponible en: http://www.cippec.org/ Main.php?do = documentsDoDownload\&id $=182$.

16. Chisari O, Estache A, Romero C. Winners and losers from utility privatization in Argentina: lessons from a general equilibrium model [Internet]. Washington DC: World Bank; 1997 [citado 7 ago
2011] WPS1824. Disponible en: http://econ.worldbank.org/external/default/main?pagePK $=64165$ 259\&theSitePK $=469372$ \&piPK $=64165421 \&$ men uPK $=64166093 \&$ entitylD $=000009265 \_397120$ 1161336

17. Esrey SA, Potash JB, Roberts L, Shiff C. Effects of improved water supply and sanitation on Ascariasis, Diarrhea, Dracunculiasis, Hookworm Infection, Schistosomiasis and Trachoma. Bulletin of the World Health Organization. 1991;69(5):609-621.

18. Auge MP, Hernández MA, Hernández L. Actualización del conocimiento del acuífero semiconfinado Puelche en la provincia de Buenos Aires, Argentina. En: Bocanegra E, Hernandez M, Usunoff E, editores. Gorundwater and human development. London: Taylor \& Francis; 2005.

19. Auge MP. Hidrología de La Plata, provincia de Buenos Aires: Relatorio XVI Congreso Geológico Argentino, La Plata, Argentina [Internet]. 2005 [citado 20 may 2012]. Disponible en: http://www.alhsud.com/public/articulos/Hidrogeolog $\%$ C3\%ADa La_Plata.pdf.

20. Auge MP, Hernández M. Características geohidrológicas de un acuífero semiconfinado (Puelche) en la Llanura Bonaerense: Su implicancia en el ciclo hidrológico de las Ilanuras dilatadas. En: Unesco, Ministerio de Obras y Servicios Públicos. Hidrología de las grandes Ilanuras: Actas del Coloquio de Olavarría, 1983. Buenos Aires: Unesco, Comité Nacional Argentino para el Programa Hidrológico Internacional; 1984.

21. Auge MP. Vulnerabilidad de Acuíferos. Revista Latinoamericana de Hidrología. 2004;(4):85-103.

\section{FORMA DE CITAR}

Monteverde M, Cipponeri M, Angelaccio C, Gianuzzi L. Origen y calidad del agua para consumo humano: salud de la población residente en el área de la cuenca Matanza-Riachuelo del Gran Buenos Aires. Salud Colectiva. 2013;9(1):53-63.

Recibido el 5 de septiembre de 2012

Versión final presentada el 25 de noviembre de 2012

Aprobado 5 de febrero de 2013 
